\title{
DECIDING EXISTENCE OF RATIONAL POINTS ON CURVES: AN EXPERIMENT
}

\author{
NILS BRUIN AND MICHAEL STOLL
}

\section{INTRODUCTION}

The problem to decide whether a given algebraic variety defined over the rational numbers has rational points is fundamental in Arithmetic Geometry. Abstracting from concrete examples, this leads to the question whether there exists an algorithm that is able to perform this task for any given variety. This is probably too much to ask for: we know that Hilbert's Tenth Problem, which asks the same question for integral points on general affine varieties, has a negative answer. But we can hope for a more favorable outcome if we restrict the class of varieties we consider.

It is then most natural to look at curves first, since they have been studied very intensely, resulting in a good theoretical knowledge and a very rich supply of algorithmic methods. Also, it makes sense to consider the geometrically nicest class of varieties, namely those that are projective. Since it is easy to check whether a curve has rational singular points, we can assume that the curve is smooth. Therefore, the question we are specifically interested in is the following.

Question 1.1. Is there an algorithm that decides for any given smooth projective curve $C / \mathbb{Q}$ whether $C$ has rational points or not?

Since we can always algorithmically prove that $C(\mathbb{Q}) \neq \emptyset$ if rational points exist by simply enumerating all rational points of the relevant projective space and checking for each point if it is on $C$, until we find a rational point on $C$, our question is equivalent to the following, seemingly more restricted version.

Question 1.2. Is there an algorithm that verifies that $C(\mathbb{Q})=\emptyset$ for any given smooth projective curve $C / \mathbb{Q}$ without rational points?

"Verification" here means that the algorithm constructs a proof of some kind.

Date: April 25, 2006.

2000 Mathematics Subject Classification. 11D41, 11G30, 11Y50 (Primary) 14G05, 14G25, 14H25, 14H45, 14Q05 (Secondary).

Research of the first author supported by NSERC. 
For curves of genus 0, our question has a positive answer since the Hasse Principle holds for these curves: a curve of genus 0 has rational points if and only if it is "everywhere locally solvable" (ELS), i.e., it has real points and $p$-adic points for all primes $p$. Since for a general curve $C$, we can check algorithmically whether it has points everywhere locally, we can assume that $C$ is ELS. The main problem is then to show that $C(\mathbb{Q})$ is empty even though $C$ is ELS.

If $C$ is a curve of genus 1 with Jacobian elliptic curve $E$, then we can perform descent calculations (on $E$ or on $C$ ), which will succeed in proving that $C(\mathbb{Q}$ ) is empty if $C$ represents an element of $\amalg(E)$ that is not divisible. In particular, if we assume that $\amalg(E)$ is finite for all elliptic curves $E / \mathbb{Q}$, then our question has a positive answer for curves of genus 1 as well.

We will therefore focus our attention to curves of higher genus. It is only since fairly recently that there is some confidence that the question might have a positive answer, spurred by progress on the theoretical side St3. St4 and also by heuristcal considerations $[\mathrm{PO}$. In this paper, we attempt to give supporting evidence of a more practical kind, by applying the available algorithms (with some new improvements and additions) to a large number of curves in order to see if we actually can decide for each of them whether they have rational points or not.

The obvious class of curves to look at for a first attempt at gathering evidence are the curves of genus 2. Their main advantage is that quite a variety of algorithms are available for them, and so we can hope to use them as adequate test cases. In order to keep the computational effort within reasonable limits, we decided to consider "small" genus 2 curves. More precisely, our initinal set of curves consists of all genus 2 curves over $\mathbb{Q}$ that have a model of the form

$$
y^{2}=f(x)=f_{6} x^{6}+f_{5} x^{5}+f_{4} x^{4}+f_{3} x^{3}+f_{2} x^{2}+f_{1} x+f_{0}
$$

with integral coefficients $f_{0}, f_{1}, \ldots, f_{6}$ satisfying $\left|f_{j}\right| \leq 3$. Excluding non-squarefree $f$ or $f$ of degree $\leq 4$ and identifying isomorphic curves, our initial set contains 196211 isomorphism classes of curves.

In Section 2, we describe our findings, and in Section 3, we give an overview of the methods we have used. The details on the new methods and the improvements on existing methods we have made can be found in a series of forthcoming papers [BS1, BS2, BS3].

A complete list of all curves considered and lists of indications on how to prove that each curve does or does not have rational points are available at [BS4].

Acknowledgments. We would like to thank Victor Flynn and Bjorn Poonen for useful discussions related to our project. M.S. thanks the Computational Laboratory for Analysis, Modeling and Visualization (CLAMV) of International University Bremen for the possibility to use computing time on the CLAMV Teaching Lab machines. This was used for substantial parts of the computations that were 
done in the course of this project. For the computations, the MAGMA [M] system was used.

\section{Results}

In a first step, we searched for a small rational point on each curve $C$. Note that $C$ has one or two obvious points if $f_{0} \in\{0,1\}$ or $f_{6} \in\{0,1\}$ ( $C$ is considered to have one or two rational points "at infinity" if $f_{6}=0$ or a non-zero square, respectively). At a later stage, we searched for larger rational points on those curves that were not yet decided. The largest points found at this stage were $\left(1519 / 601,4816728814 / 601^{3}\right)$ on

$$
C: y^{2}=3 x^{6}-2 x^{5}-2 x^{4}-x^{2}+3 x-3
$$

and $\left(193 / 436,165847285 / 436^{3}\right)$ on

$$
C: y^{2}=3 x^{6}-3 x^{5}-x^{4}-x^{3}-3 x^{2}+x-3 .
$$

This left us with 58681 curves $C$ without (apparent) rational points, for which we need to prove that $C(\mathbb{Q})=\emptyset$. Among these, there are 29278 curves with points everywhere locally. Together with the curves that do have rational points, this means we found 166808 with points everywhere locally, which is about $85 \%$ of all the curves we considered. In PSt2 (see also PSt1, Section 9), it is shown that the set of polynomials $f$ giving rise to an everywhere locally solvable curve has a well-defined, positive density $\delta$. Numerical estimates of the local densities involved lead to a value close to 0.85 for $\delta$, which fits well with our observations.

The next stage in the procedure is to perform a 2-cover descent on each of the remaining curves. This constructs (implicitly) a finite collection of curves $D_{j}$ that cover $C$ and such that every rational point on $C$ is the image of a rational point on some $D_{j}$. So if we obtain an empty covering collection $\left\{D_{j}\right\}$, this proves that $C$ has no rational points. For a more precise description of the computation, see Section 3.1. With this method, we were able to prove that $C(\mathbb{Q})$ is empty for all but 1492 curves.

For these 1492 curves, we wanted to perform a "Mordell-Weil sieve" computation. The idea is as follows. Let $J$ be the Jacobian variety of $C$, and assume that we can embed $C$ into $J$. Assume also that we can determine generators of the Mordell-Weil group $J(\mathbb{Q})$. Now consider the following commutative diagram.

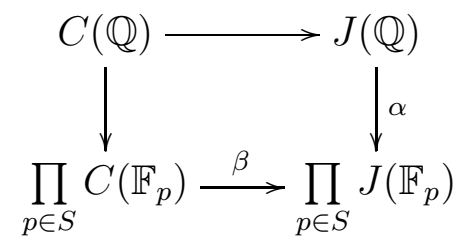


Here $S$ is some finite set of primes (of good reduction for $C$, say). Since we know $J(\mathbb{Q})$ and can find the finite sets $C\left(\mathbb{F}_{p}\right)$, we can compute the images of $\alpha$ and $\beta$. If these images do not meet, this proves that $C(\mathbb{Q})$ is empty.

As a first step, we had to find generators of the Mordell-Weil group. To do this, we first perform a 2-descent (see [St1] ) on $J$ to get an upper bound for the rank of the finitely generated abelian group $J(\mathbb{Q})$. Then we need to find the correct number of independent points in $J(\mathbb{Q})$. In order to be able to do this successfully, we had to come up with new strategies, involving a search for points on (quotients) of 2-covering spaces for $J$. See Section 3.2 for more details. In this way, we were able to find generators of a finite index subgroup of $J(\mathbb{Q})$ for all but 47 curves. It is then a fairly easy matter to check that we actually had generators of $J(\mathbb{Q})$ (modulo torsion), see St2. In the course of these computations, we also found a rational point on the 2-covering space $\mathrm{Pic}_{C}^{1}$ for $J$, which provides an embedding of $C$ into $J$. So for these 1445 curves ( 3 of rank 0, 516 of rank 1, 772 of rank 2, 152 of rank 3, and 2 of rank 4), the assumptions for the application of the MordellWeil sieve are satisfied. After several improvements of the algorithm performing the actual sieve computation (the problem here is combinatorial explosion), we were finally able to run the procedure successfully for all these curves. With the current version of the algorithm, the maximal computation time for a single curve was roughly 16 hours on a $1.7 \mathrm{GHz}$ processor; this curve is one of the two with Mordell-Weil rank 4.

For the remaining 47 curves (36 of Selmer rank 2, 10 of Selmer rank 3, and one of Selmer rank 4), the number of independent points we found fell short by 2 of the Selmer rank. Therefore we suspect that there is nontrivial 2-torsion in $\amalg(J)$ in these cases. In 5 out of the 10 Selmer rank 3 cases, we found a rational point on $\operatorname{Pic}_{C}^{1}$. Here we expect that $\amalg(J)[2]=(\mathbb{Z} / 2 \mathbb{Z})^{2}$. This was confirmed by an adhoc visualization argument. See [Br2, BF2] for a description and detailed analysis of this method for hyperelliptic curves with a rational branch point.

For these 5 curves, we know that $\operatorname{rank} J(\mathbb{Q})=1$, and we have an embedding of $C$ into $J$, so that we can run the Mordell-Weil sieve procedure, which confirms that there are no rational points on these curves.

One example we found is

$$
C: y^{2}=f(x)=-x^{6}+2 x^{5}+3 x^{4}+2 x^{3}-x-3 .
$$

When we consider a quadratic twist of this curve,

$$
C^{(-1)}: y^{2}=-f(x),
$$

we find that $J^{(-1)}(\mathbb{Q})$ is of rank 4 , where $J^{(-1)}$ is the Jacobian of $C^{(-1)}$. A slightly more involved computation gives that $J(\mathbb{Q}(\sqrt{-1}))$ is of rank at most 5 . Since this rank is the sum of the ranks of $J(\mathbb{Q})$ and $J^{(-1)}(\mathbb{Q})$, this means that the rank of 
$J(\mathbb{Q})$ can be at most 1 . This is less than the rank bound 3 we obtain from a 2-descent on $J$ directly.

In the remaining 42 cases, we did not find a rational point on $\mathrm{Pic}_{C}^{1}$. On the other hand, from the 2-cover descent, we know that $C$ has everywhere locally solvable 2 -coverings; the same must then be true for $\mathrm{Pic}_{C}^{1}$. This means that the class of $\operatorname{Pic}_{C}^{1}$ in $\amalg(J)$ is divisible by 2 . If $\operatorname{Pic}_{C}^{1}(\mathbb{Q})=\emptyset$, then this implies that there are elements of order 4 in $\amalg(J)$. The computations necessary for a visualization argument are hardly feasible in this situation: one needs to compute the 2-Selmer group of $J$ over a quartic number field. This involves finding an $S$-unit group in a degree 24 number field.

Still, assuming GRH, we were successful for 4 curves in showing that the true Mordell-Weil rank is smaller than the bound obtained from a 2-descent. One of these curves is

$$
C: y^{2}=-3 x^{6}-x^{5}+2 x^{4}+2 x^{2}-3 x-3 .
$$

The Jacobians of the quadratic twists by $2,-3,-6$ can easily be shown to have Mordell-Weil ranks 4, 4,3 respectively. Furthermore, a 2-descent shows, conditional on $\mathrm{GRH}$, that $J(\mathbb{Q}(\sqrt{2}, \sqrt{-3}))$ is of rank at most 11. It follows that $J(\mathbb{Q})$ must be of rank 0 .

We do not expect that results along these lines can be extended much further. To complement the above computations, we computed the analytic order of $\amalg(J)$. For this we had to assume that the $L$-series $L(C, s)$ can be analytically continued and satisfies the usual functional equation. The results of our computations are consistent with this assumption. First of all, we verified that the $r$-th derivative of $L(C, s)$ at $s=1$ is nonzero, where $r$ is the conjectured rank of $J(\mathbb{Q})$ (i.e., the number of independent points we have found). Secondly, the analytic order of $\amalg(J)$ comes out to be 16 for the 42 curves where we expect elements of order 4 , and it is 4 for the 5 curves mentioned above, where we expect $\amalg(J)=(\mathbb{Z} / 2 \mathbb{Z})^{2}$. Hence, assuming standard conjectures on $L$-series and the Birch and SwinnertonDyer Conjecture, we find that $\operatorname{Pic}_{C}^{1}(\mathbb{Q})=\emptyset$ for our 42 curves, and therefore $C(\mathbb{Q})=$ $\emptyset$ as well.

The main result of our experiment is that we were successful in deciding the existence of rational points unconditionally on all but 42 of our curves. If we assume standard conjectures, we can prove that there are no rational points on these remaining 42 curves as well. This very positive result lends strong support to the belief that existence of rational points on curves should be decidable, at least for curves of genus 2 .

Note that our results also provide evidence for the conjecture that the BrauerManin obstruction should be the only obstruction against rational points on curves. For all but the 1492 curves surviving a 2-cover descent, we verify this unconditionally. For the remaining curves, we need to assume that $\amalg(J)$ has 


\begin{tabular}{|l|r|r|}
\hline All curves & 196211 & $100.00 \%$ \\
Curves with rational points & 137530 & $70.09 \%$ \\
Curves without rational points & 58681 & $29.91 \%$ \\
ELS curves total & 166808 & $85.01 \%$ \\
ELS curves without rational points & 29278 & $14.92 \%$ \\
Curves with ELS 2-covers among these & 1492 & $0.76 \%$ \\
Curves that need GRH or BSD conjecture & 42 & $0.00 \%$ \\
\hline
\end{tabular}

TABLE 1. Curve statistics (ELS = everywhere locally solvable)

\begin{tabular}{|l||r|r|r||r|}
\hline $\operatorname{conj} . \amalg(J)$ & 0 & $(\mathbb{Z} / 2 \mathbb{Z})^{2}$ & $(\mathbb{Z} / 4 \mathbb{Z})^{2}$ & Total \\
\hline $\operatorname{rank} J(\mathbb{Q})=0$ & 3 & & 36 & 39 \\
$\operatorname{rank} J(\mathbb{Q})=1$ & 516 & 5 & 5 & 526 \\
$\operatorname{rank} J(\mathbb{Q})=2$ & 772 & & 1 & 773 \\
$\operatorname{rank} J(\mathbb{Q})=3$ & 152 & & & 152 \\
$\operatorname{rank} J(\mathbb{Q})=4$ & 2 & & & 2 \\
\hline all ranks & 1445 & 5 & 42 & 1492 \\
\hline
\end{tabular}

TABLE 2. Ranks and conjectural $\amalg$ for the curves surviving 2-cover descent

trivial divisible subgroup, plus whatever assumptions were necessary in addition for individual curves. See [Sc, [F], St3] for details on how our computations relate to the Brauer-Manin obstruction.

\section{Methods}

In this section, we give an overview of the methods we have used. Detailed descriptions will be provided in [BS1, BS2, BS3].

3.1. 2-Cover Descent. Let the curve $C$ be given by the equation $y^{2}=f(x)$, and let $L$ denote the étale $\mathbb{Q}$-algebra $\mathbb{Q}[T] /(f(T))$. We let $\theta$ be the image of $T$ in $L$. If $f$ has a rational root or is of odd degree then $C$ has a rational point. Therefore, we can assume in the following that $f$ is of degree 6 and has no rational roots. Let $a$ be the leading coefficient of $f$. Let $k=\mathbb{Q}$ or $k=\mathbb{Q}_{v}$, where $v$ is a prime $p$ or $\infty$ and $\mathbb{Q}_{\infty}=\mathbb{R}$. We have a map

$$
F: C(k) \longrightarrow \frac{\left(L \otimes_{\mathbb{Q}} k\right)^{*}}{k^{*}\left(L \otimes_{\mathbb{Q}} k\right)^{* 2}}, \quad P=(x, y) \longmapsto(x-\theta) \cdot k^{*}\left(L \otimes_{\mathbb{Q}} k\right)^{* 2},
$$


whose image is contained in the subset of elements whose norm in $k^{*} / k^{* 2}$ is the class of $a$. Note that $y^{2}=f(x)=a N_{L \otimes k / k}(x-\theta)$.

As in the case of 2 -descent on the Jacobian $J$ of $C$, one shows that $F\left(C\left(\mathbb{Q}_{p}\right)\right)$ is contained in the image of the $p$-adic units for all odd $p$ not dividing the discriminant of $f$ (see [St1]). Let $H^{\prime} \subset L^{*} / \mathbb{Q}^{*} L^{* 2}$ be the (finite) set of elements that come from $p$-adic units for this set of primes, and let $H \subset H^{\prime}$ be the subset of elements whose norm is $a \mathbb{Q}^{* 2}$; then $F(C(\mathbb{Q})) \subset H$. There are cases when $H$ is already the empty set; we can then immediately conclude that $C(\mathbb{Q})=\emptyset$. An example of this is

$$
C: y^{2}=2 x^{6}+3 x^{5}+x^{4}-3 x^{3}-2 x^{2}+2 x+3 \text {. }
$$

For this curve it can be checked that $2 \mathbb{Q}^{* 2}$ is not the norm of an element of $H^{\prime}$.

We denote $L \otimes_{\mathbb{Q}} \mathbb{Q}_{v}$ by $L_{v}$. Let $H_{v} \subset L_{v}^{*} / \mathbb{Q}_{v}^{*} L_{v}^{* 2}$ denote the subset of elements whose norm is $a \mathbb{Q}_{v}^{* 2}$. We have the following commutative diagram.

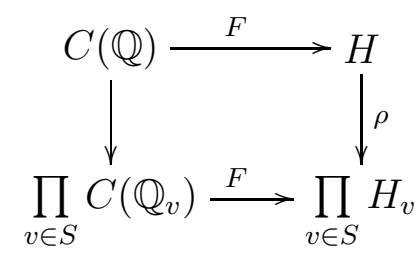

Here, $S$ is a suitable finite set of places. One can show that $F\left(C\left(\mathbb{Q}_{p}\right)\right)=H_{p}$ for $p>1154$ if $p$ does not divide $\operatorname{disc}(f)$. Therefore, we obtain the maximal amount of information when we choose

$$
S=\{\infty\} \cup\{p: p<1154\} \cup\{p: p \mid \operatorname{disc}(f)\} .
$$

Note that the sets $H_{v}$ are finite and that $F$ is $v$-adically continuous, hence locally constant. Therefore we can compute $F\left(C\left(\mathbb{Q}_{v}\right)\right) \subset H_{v}$ explicitly for every $v$. Following [PSc, we define the fake 2-Selmer set of $C, \operatorname{Sel}_{\text {fake }}^{(2)}(C)$, to be the preimage in $H$ under $\rho$ of the image of the lower $F$ map. Then $F$ maps $C(\mathbb{Q})$ into $\operatorname{Sel}_{\text {fake }}^{(2)}(C)$, hence if $\operatorname{Sel}_{\text {fake }}^{(2)}(C)=\emptyset$, then we know that $C(\mathbb{Q})$ is empty as well.

The geometric interpretation of the elements of $\operatorname{Sel}_{\text {fake }}^{(2)}(C)$ is that they correspond to everywhere locally solvable 2-covering curves of $C$. If $\xi \in L$ represents an element of $\operatorname{Sel}_{\text {fake }}^{(2)}(C)$, then the corresponding covering $D_{\xi} \rightarrow C$ can be obtained as follows. We write $z=z_{0}+z_{1} \theta+\cdots+z_{5} \theta^{5}$ for a generic element of $L$. The condition for a rational point $P=(x, y)$ on $C$ to be in the image of $D_{\xi}(\mathbb{Q})$ is that

$$
(x-\theta) \cdot \mathbb{Q}^{*} L^{* 2}=F(P)=\xi \cdot \mathbb{Q}^{*} L^{* 2} .
$$

So $x-\theta=c \xi z^{2}$ for some $c \in \mathbb{Q}, z \in L$. Expanding the right hand side in terms of powers of $\theta$, we obtain four quadrics in the six variables $z_{0}, \ldots, z_{5}$ that express the condition that the coefficients of $\theta^{2}, \ldots, \theta^{5}$ have to vanish. These four quadrics define the curve $D_{\xi} \subset \mathbb{P}^{5}$ of degree 16 and genus 17 . To obtain the covering map, note that $x$ can be recovered from the coefficients of 1 and $\theta$ in $\xi z^{2}$, and $y$ can 
be recovered from these, the norm of $z$ and a square root of $N(\xi) / a$. One has to make a sign choice here, so that there are really two different covering maps in most cases. See also Br1, 5.3] and BF1 for a description of the cover. For details on how to compute $\operatorname{Sel}_{\text {fake }}^{(2)}(C)$ efficiently, see [BS1].

3.2. Finding Generators. Since the simplest generally available model of the Jacobian $J$ is given by 72 quadrics in $\mathbb{P}^{15}$ (see $[\mathrm{CF}$ ), it is usually not a good idea to search for rational points directly on $J$. A better alternative is to consider the Kummer surface $K=J /\{ \pm 1\}$, which sits naturally as a quartic surface in $\mathbb{P}^{3}$. We now can search for rational points on $K$ that lift to rational points on $J$. A fairly efficient implementation of this idea that uses mod- $p$ information for several primes $p$ in order to rule out many candidates is obtainable as $j$-points from M. Stoll's homepage; this program is also incorporated in MAGMA. This approach is feasible for points of naive (non-logarithmic) height around 10000 or a little bit more. (The height is that of the image point on $K \subset \mathbb{P}^{3}$.)

However, there are many cases in our list where there is a much bigger generator. In order to find these, we use the idea (by now in common use in the context of elliptic curves) that rational points on $J$ lift to rational points on a 2-covering of $J$ that should be much smaller. Therefore we attempt to search for rational points on these 2-coverings. However, these coverings are as complicated geometrically as $J$ itself, therefore we consider a suitable quotient again.

Recall (see PSc, St1) that the fake 2-Selmer group of $J$ is a finite subgroup of $L^{*} / \mathbb{Q}^{*} L^{* 2}$. It contains the image of $J(\mathbb{Q})$ under a map that sends a rational point $P$ to an element represented by $x_{0}-x_{1} \theta+\theta^{2} \in L$, for certain $x_{0}, x_{1} \in \mathbb{Q}$ depending on $P$. Let $\xi$ be an element of the fake Selmer group. We use the same idea as in the previous section to construct a surface $K_{\xi}$ : we are looking for $z \in L$ such that $\xi z^{2}$ does not involve $\theta^{3}, \theta^{4}, \theta^{5}$. This gives us an intersection $K_{\xi}$ of three quadrics in $\mathbb{P}^{5}$. We simplify the defining equations as far as possible by a change of projective coordinates so that they have small coefficients. Then we perform a search for rational points on $K_{\xi}$ using a $p$-adic variant of Elkies' lattice-based point searching techniques (see [El]). For each point found, we check whether it corresponds to a rational point on $J$. In this way, we can find points in $J(\mathbb{Q})$ whose image in the fake Selmer group is nontrivial.

However, note that $\operatorname{Pic}_{C}^{1}$ is a 2 -covering of $J$ via the map $D \mapsto 2 D-W$, where $D \in \operatorname{Pic}_{C}^{1}$ and $W$ is the canonical class. Its image in the fake Selmer group is trivial, so the method above will not help in finding rational points on it. Instead, in analogy to the use of the Kummer surface when searching for points on $J$, we can use the dual Kummer surface (see CF], Chapter 4). We can even go a step further and consider 2-coverings of $\mathrm{Pic}_{C}^{1}$. In this case, we obtain 3-dimensional varieties, given as intersections of two quadrics in $\mathbb{P}^{5}$, that are quotients of $\mathbb{P}^{1}$ bundles over the coverings we are interested in. We can search for rational points 
on these 3 -folds and check whether they give rise to a rational point on $J$. This amounts to a partial explicit 4-descent on $J$. It is therefore perhaps not surprising that we were able to find some quite large generators in this way. The record example is

$$
C: y^{2}=-3 x^{6}+x^{5}-2 x^{4}-2 x^{2}+2 x+3
$$

with $J(\mathbb{Q})$ infinite cyclic generated by $P_{1}+P_{2}-W$ where the $x$-coordinates of $P_{1}$ and $P_{2}$ are the roots of

$$
x^{2}+\frac{37482925498065820078878366248457300623}{34011049811816647384141492487717524243} x+\frac{581452628280824306698926561618393967033}{544176796989066358146263879803480387888} ;
$$

the canonical logarithmic height of this generator is 95.26287. The second largest example is

$$
C: y^{2}=-2 x^{6}-3 x^{5}+x^{4}+3 x^{3}+3 x^{2}+3 x-3
$$

with $J(\mathbb{Q})$ generated by a point coming from

$$
x^{2}+\frac{83628354341362562860799153063}{26779811954352295849143614059} x+\frac{852972547276507286513269157689}{321357743452227550189723368708} .
$$

The canonical height of this generator is 77.33265 .

For details see [BS2].

3.3. Mordell-Weil Sieve. As mentioned in Section 2, we consider the commutative diagram

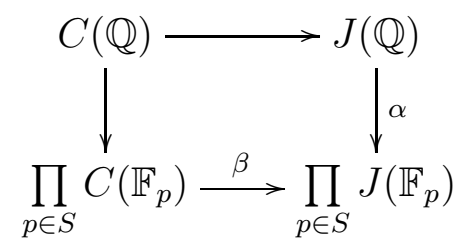

with a suitable finite set $S$ of (good) primes. In some cases, it can be helpful to use some more general finite quotient of $J\left(\mathbb{Q}_{p}\right)$ instead of $J\left(\mathbb{F}_{p}\right)$, for example to make use of information modulo higher powers of $p$, or also in order to use information at primes of bad reduction. In the following discussion, we will assume for simplicity that we are working with $J\left(\mathbb{F}_{p}\right)$.

Our goal is to prove that the images of $\alpha$ and $\beta$ above do not meet, for some set $S$, which implies that $C(\mathbb{Q})=\emptyset$. This approach was (to our knowledge) first suggested by Scharaschkin [Sc]. Flynn [F] used it for more extensive calculations. We would like to mention here that in the course of improving the algorithms, we were able to prove all the curves marked "Unresolved" in the tables of [F] to have no rational points. (All but five of these already succumb to a 2-cover descent, the remaining five, all of which have Jacobians of Mordell-Weil rank 3, can be dealt with using our Mordell-Weil sieve implementation.)

The basic algorithmic problem one has in this computation is that the product of the $J\left(\mathbb{F}_{p}\right)$ can be a very large group. One approach to keep the combinatorics in check is to work with $J(\mathbb{Q}) / B J(\mathbb{Q})$ and $\prod_{p} J\left(\mathbb{F}_{p}\right) / B J\left(\mathbb{F}_{p}\right)$ for a suitable choice 
of $B$. In practice, we compute the subset of $J(\mathbb{Q}) / B J(\mathbb{Q})$ that maps under $\alpha$ into the image of $\beta$. We first need to choose a promising set $S$ of primes. Since we can only hope to arrive at a contradiction when the group orders of the $J\left(\mathbb{F}_{p}\right)$ have (preferably large) common factors, we select those primes $p$ for which the order of $J\left(\mathbb{F}_{p}\right)$ is sufficiently smooth. We then compute the image of $C\left(\mathbb{F}_{p}\right)$ in $J\left(\mathbb{F}_{p}\right)$ and the image of the generators of $J(\mathbb{Q})$. Note that this involves a discrete logarithm computation in $J\left(\mathbb{F}_{p}\right)$ for each point in $C\left(\mathbb{F}_{p}\right)$ and each generator of $J(\mathbb{Q})$. While this is a hard problem in general, it is harmless here since the group order is smooth and we can reduce to several discrete logs in small groups.

In the following discussion, the set $S$ is fixed. For a given $B$, we can find the image $C_{B, p}$ of $C\left(\mathbb{F}_{p}\right)$ in $J\left(\mathbb{F}_{p}\right) / B J\left(\mathbb{F}_{p}\right)$, and we can then compute the expected size

$$
n(B)=\#(J(\mathbb{Q}) / B J(\mathbb{Q})) \prod_{p \in S} \frac{\# C_{B, p}}{\#\left(J\left(\mathbb{F}_{p}\right) / B J\left(\mathbb{F}_{p}\right)\right)}
$$

of the subset $A(B)$ of $J(\mathbb{Q}) / B J(\mathbb{Q})$ that maps into these images for all $p \in S$. We now search for a sequence $1=B_{0}, B_{1}, \ldots, B_{m}$ such that $B_{j+1}=B_{j} q_{j}$ for some prime $q_{j}$, such that $n\left(B_{m}\right) \ll 1$ and such that $\max _{j} n\left(B_{j}\right)$ is not too large. (See $[\mathrm{Po}$ for heuristics why there should exist $B$ with $n(B) \ll 1$, at least when $S$ is sufficiently large.)

After we have fixed our sequence $\left(B_{j}\right)$, we successively compute the sets $A\left(B_{j}\right)$ for $j=1,2, \ldots$ until $A\left(B_{j}\right)=\emptyset$. If we reach $j=m$ and $A\left(B_{m}\right) \neq \emptyset$, then we can check if this is caused by a exhibitable rational point. The set $A\left(B_{m}\right)$ will give a very good indication of which elements of $J(\mathbb{Q})$ could give rise to such a point. If we cannot find a point, we can extend the sequence or choose a bigger set $S$. In fact, this situation never occurred in our computations.

To obtain $A\left(B_{j+1}\right)$ from $A\left(B_{j}\right)$, we run through the elements of $A\left(B_{j}\right)$. For each element, we run through its possible lifts to $J(\mathbb{Q}) / B_{j+1} J(\mathbb{Q})$, and for each lift check whether it maps into the image of $C \bmod p$ for all relevant $p$ (i.e., such that the largest power of $q_{j+1}$ dividing $B_{j+1}$ also divides the exponent of $J\left(\mathbb{F}_{p}\right)$ ). The largest set $A\left(B_{j}\right)$ that we encountered in our computations had a size of about $10^{6}$. It is perhaps worth while mentioning that the estimate $n(B)$ for $\# A(B)$ was in most cases accurate up to a factor of 2 to 5 , so that a value $n\left(B_{m}\right)<10^{-3}$ (say) virtually guarantees success in practice.

For details see [BS3].

3.4. BSD Computations. Finally, let us give some indications of how to compute the analytic order of $\amalg$. Dokchitser [Do] describes how the numbers $L^{(r)}(C, 1)$ can be computed numerically, given (i) the coefficients $a_{n}$ of the $L$-series for sufficiently many $n$, (ii) the conductor $N$ of $C$ (or $J$ ), and (iii) the sign $\varepsilon$ in the (conjectured) functional equation. The latter is determined by the parity of the rank. The coefficients $a_{p}$ and $a_{p^{2}}$ for good primes $p$ can be computed by counting 
the points in $C\left(\mathbb{F}_{p}\right)$ and $C\left(\mathbb{F}_{p^{2}}\right)$; these coefficients then determine $a_{p^{k}}$ for all $k \geq 1$. For bad primes $p$, the coefficients can be deduced from a minimal proper regular model of $C$ over $\mathbb{Z}_{p}$; a description of the computation of such a model can be found in $\mathrm{F}+$. The most frequent case is that an odd prime $p$ divides the discriminant of the polynomial $f$ just once; then

$$
f(x) \equiv(x-a)^{2} g(x) \bmod p,
$$

and the Euler factor at $p$ of $L(C, s)$ depends on whether $g(a)$ is a square or not and the number of $\mathbb{F}_{p}$-points on the genus 1 curve $y^{2}=g(x)$. In most other cases, the original model is already regular. For all of the curves, we computed $5 \cdot 10^{5}$ or even $10^{6}$ coefficients; this led to an error in the value of \#Ш $(J)$ predicted by the Birch and Swinnerton-Dyer Conjecture of less than $10^{-3}$ in all cases.

We can find the odd part of the conductor $N$ using Q. Liu's genus2reduction program [Li2, based on Li1]. If the given model of the curve is regular at 2, then the power of 2 dividing $N$ is that dividing the discriminant of $C$. Otherwise, we use the approach described in [Do, $\S 7$ ] to determine the right power of 2 (which is then less than that in the discriminant). We can then verify the functional equation for the inverse Mellin transform of $L(C, s)$ numerically, thus corroborating our computations.

Given the value of $L^{(r)}(C, 1)$, we compute the analytic order of $\amalg$ by solving the conjectural equality between $L^{(r)}(C, 1) / r$ ! and a combination of invariants of $C$ and $J$ for \#W. See $\mathrm{F}+$ for how to compute the other invariants. As already mentioned, the values we obtain were always close to an integer, which was 4 in the five cases where we expect $\amalg(J) \cong(\mathbb{Z} / 2 \mathbb{Z})^{2}$ and 16 in the remaining cases, where we expect $\amalg(J) \cong(\mathbb{Z} / 4 \mathbb{Z})^{2}$.

\section{REFERENCES}

[Br1] N. BRuIn: Chabauty methods and covering techniques applied to generalized Fermat equations, CWI Tract 133, 77 pages (2002).

[Br2] N. Bruin: Visualisation of Sha[2] in Abelian Surfaces, Math. Comp. 73, no. 247, 14591476 (2004).

[BF1] N. BRuin and E.V. Flynn: Towers of 2-covers of hyperelliptic curves, Trans. Amer. Math. Soc. 357, 4329-4347 (2005).

[BF2] N. BRuin and E.V. Flynn: Exhibiting Sha[2] on Hyperelliptic Jacobians, to appear in Journal of Number Theory (2005?).

[BS1] N. BRUin and M. StolL: 2-cover descent on hyperelliptic curves, in preparation.

[BS2] N. Bruin and M. Stoll: Finding Mordell-Weil generators on genus 2 Jacobians, in preparation.

[BS3] N. Bruin and M. Stoll: The Mordell-Weil sieve: Proving non-existence of rational points on curves, in preparation.

[BS4] N. BRuin and M. StolL: Electronic data, available from http://www.cecm.sfu.ca/ nbruin/smallgenus2curves. 
[CF] J.W.S. Cassels and E.V. Flynn: Prolegomena to a middlebrow arithmetic of curves of genus 2, Cambridge University Press, Cambridge (1996).

[Do] T. Dokchitser: Computing special values of motivic L-functions, Exp. Math. 13, No.2, 137-149 (2004).

[El] N. D. ElKIES: Rational points near curves and small nonzero $\left|x^{3}-y^{2}\right|$ via lattice reduction, in: W. Bosma (ed.): Algorithmic number theory (Leiden, 2000), Springer, Berlin, Lecture Notes in Comput. Sci. 1838, 33-63 (2000).

[Fl] E.V. Flynn: The Hasse Principle and the Brauer-Manin obstruction for curves, Manuscripta Math. 115, 437-466 (2004).

[F+] E.V. Flynn, F. Leprévost, E.F. Schaefer, W.A. Stein, M. Stoll and J.L. Wetherell: Empirical evidence for the Birch and Swinnerton-Dyer conjectures for modular Jacobians of genus 2 curves, Math. Comp. 70, 1675-1697 (2001).

[Li1] Q. LIU: Conducteur et discriminant minimal de courbes de genre 2, Compositio Math. 94, 51-79 (1994).

[Li2] Q. LiU: genus2reduction program, available at http://www.math.u-bordeaux.fr/ liu/G2R/.

[M] MAGMA is described in W. Bosma, J. Cannon and C. Playoust: The Magma algebra system I: The user language, J. Symb. Comp. 24, 235-265 (1997). (Also see the Magma home page at http://www.maths.usyd.edu.au:8000/u/magma/.)

[Po] B. Poonen: Heuristics for the Brauer-Manin obstruction for curves, Preprint (2005).

[PSc] B. PoOnen and E.F. SchaEfER: Explicit descent for Jacobians of cyclic covers of the projective line, J. reine angew. Math. 488, 141-188 (1997).

[PSt1] B. Poonen and M. Stoll: The Cassels-Tate pairing on principally polarized abelian varieties, Ann. of Math. 150, 1109-1149 (1999).

[PSt2] B. Poonen and M. Stoll: A local-global principle for densities, in: Scott D. Ahlgren (ed.) et al.: Topics in number theory. In honor of B. Gordon and S. Chowla. Kluwer Academic Publishers, Dordrecht. Math. Appl., Dordr. 467, 241-244 (1999).

[Sc] V. Scharaschkin: The Brauer-Manin obstruction for curves, Manuscript.

[St1] M. StolL: Implementing 2-descent on Jacobians of hyperelliptic curves, Acta Arith. 98, 245-277 (2001).

[St2] M. StolL: On the height constant for curves of genus two, II, Acta Arith. 104, 165-182 (2002).

[St3] M. Stoll: Finite descent and rational points on curves, Preprint (2006).

[St4] M. StolL: Finite coverings and rational points, Oberwolfach Report 32/2005 (2005).

Department of Mathematics, Simon Fraser University, Burnaby, BC, Canada V5A $1 \mathrm{~S} 6$

E-mail address: nbruin@cecm.sfu.ca

School of Engineering and Science, International University Bremen, P.O.Box 750561, 28725 Bremen, Germany.

E-mail address: m.stoll@iu-bremen.de 